\title{
Neues aus der Literatur
}

\section{Weihrauch und Myrrhe}

\section{- Kulturgeschichte und wirtschaftliche Bedeutung Botanik - Chemie - Medizin}

Autoren: D. Martinetz, K. Lohs, J. Janzen

Verlag: Wissenschaftliche Verlagsgesellschaft Stuttgart 1989. 236 S., 92 Abb. (WVG-Bildatlas); gebunden DM 48,-. ISBN 3-8047-1019-9 (WVG). Die Originalausgabe erschien unter dem Titel „Weihrauch und Myrrhe/Kostbarkeiten der Vergangenheit im Licht der Gegenwart" im Akademie-Verlag Berlin 1989

Ein Naturstoff macht Geschichte. Ein Harz bestimmt politische Entwicklungen ganzer Epochen und Kulturen. Legenden spinnen sich um Gewinnung und Handel eines Rohstoffes, dessen hervorragende Bedeutung für unsere Kulturgeschichte aus heutiger Sicht nicht einfach zu verstehen ist.

Der Einstieg in ein komplexes Thema, die Psychologie des Geruchserlebens im Zusammenhang mit der historischen Bedeutung der beiden Harze, gelingt den Autoren auf eine ganz unerwartete Weise. Sozusagen „kontrapunktisch", wie sie selber schreiben, untersetzen sie dabei das kulturhistorische Bild des Weihrauchs und der Myrrhe mit der Umweltproblematik unserer Tage.

In fesselnder Folge geht es dann durch die Botanik der beiden Stammpflanzen, Boswellia und Commiphora, über ihre Bedeutung in der Medizin weiter zur Chemie ihrer Inhaltsstoffe. Gut verständlich und übersichtlich, mit vielen Hinweisen auf den historischen Kontext, wird dem Fachmann und auch dem interessierten Laien Einblick in die faszinierende Welt dieser beiden Harzdrogen geboten. Die analytischen Resultate sind auf dem neuesten Stand und werden manchen interessieren, der mit ätherischen Ölen, Harzen, Balsamen, Aromastoffen und Essenzen gearbeitet hat.

Ein großes Kapitel über die ökonomischen, sozialen und soziokulturellen Aspekte der heutigen Harzsammelwirtschaft in den klassischen Produktionsgebieten, in Südarabien und am Horn von Afrika, vervollständigen diese Arbeit und machen aus dem reich illustrierten und sehr schön bebilderten Buch ein Übersichtswerk über ein sehr spannendes Thema.

M. KESSLER (Basel)
D. Johann SCHROEDER meinte 1686, daß Weihrauch das Gedächtnis stärke, „wann man des Morgens/Mittags und Abends/ wann der Mond im Scorpion steht/ 3 Kernlein einnimmet."

Man wünschte sich zu diesem Buch die „Kernlein“ gleich als Beigabe dazu. So gelehrt und bildend, so fachwissenschaftlich präzise und kulturgeschichtlich seriös behandeln die Autoren ihr Thema, daß man diese Belehrung gern bewahren möchte. Neben einer ausführlichen, nach geographischen Regionen differenzierenden Einleitung über die heutige Verbreitung von Weihrauch und Myrrhe, behandeln die Autoren zunächst die Bedeutung von Balsamen, Harzen und Räucherwerk in der Kulturgeschichte.

Ein Kapitel über die pharmakologischen Eigenschaften verfolgt die Bedeutung von Myrrhe und Weihrauch in vergleichender Betrachtung durch die Zeitalter. Kapitel zu Chemie und den Wirkstoffen schließen sich an. Ein sozial- und wirtschaftsgeschichtlicher Exkurs über Tradition und Wandel in den Weihrauch- und Myrrhe-Sammelwirtschaften bildet den Abschluß.

Der reich und farbig bebilderte Band ist eine gelungene Synthese von natur- und geisteswissenschaftlichen Zugängen zum Thema.
Die heute im Vordergrund stehende Bedeutung des Weihrauchs als Räucherwerk läßt sich unschwer mit der Drogenwirkung in Verbindung bringen. Insofern ist es ein ganz klein wenig zu bedauern, wenn die Autoren gerade die Sozialgeschichte des Duftes nicht mit der sonstigen Tiefe des Buches behandeln. A. CORBIN, dem wir „Eine Geschichte des Geruches" verdanken, wurde nicht einbezogen. Dies ist um so erstaunlicher, als im Kapitel über die „Physiologie und Psychologie des Geruchssinns" herbe Kritik an Menschen geübt wird („Öko-Fanatiker"), deren Geruchstoleranzen anders gelagert sind als die der Autoren. Irgendwo zwischen VESPASIANS "Non olet" und den Bemühungen der ersten „Umweltkommission" (1285 zur Befreiung Londons vom Steinkohlenrauch) wird wohl die Wahrheit liegen. Auch bei Myrrhe und Weihrauch gilt in Anlehnung an PARACELSUS, daß die Menge den Geruch mache.

Mir hat es sonst sehr gut gefallen.

B. HerRmanN (Göttingen) 